\title{
Artificial neural network software for real-time estimation of olive oil qualitative parameters during continuous extraction
}

\author{
Rocco Furferi, Monica Carfagni, Marco Daou
}

\begin{abstract}
This work provides a software based on the combination of a artificial neural network (ANN) approach and a numerical method (called "refining algorithm") for the estimation of the acidity level and of peroxides number of olive oil extracted by a continuous extraction process. The estimation is achieved through the measurement of some agronomical and technological parameters commonly measured by the technicians working at the oil mills. The ANN based approach is able to perform a rough prediction of the two parameters; this prediction is then refined by means of the numerical approach. The devised software, developed using a database of experimental data collected in the years 1997-2004 in the Tuscany Region (Italy), is able to estimate the two parameters during the continuous oil extraction, thus allowing a realtime control of the oil quality during the extraction process. The experimental data are related to different kinds of extraction systems: the data collected before 2003 are mainly referred to three phases decanting while the one collected in years 2003-2004, are referred to a two phases decanter. The goal of this approach is to build up a fast and reliable quality control without performing the standard laboratory analysis. The results of the estimation achieved by the ANN based system are compared with the results of the chemical analyses carried out by Florence Commerce Chamber "Laboratorio Chimico MerceologicoAzienda Speciale CCIAA di Firenze" according in force to European Union Rules standards.

The mean error in estimation of the parameters with the combination of the ANN approach and the refining algorithm, in comparison with the standard chemical analysis, is in the range $6-8 \%$ for an oil extraction with a three phases decanting system and about $6-7 \%$ for an oil extraction with a two phases decanting system. The maximum error affecting the method (ANN + Refining) described in this work is about $15 \%$ for the first case and about $11 \%$ for the second one. The system developed in the present work is actually running on the oil mill "TEM Toscana Enologica Mori" of Florence, Italy.

The present work has been financed by the Tuscany Regional Agricultural Development and Innovation Office (ARSIA: Azienda Regionale per lo Sviluppo e I'Innovazione dell'Agricoltura) and is a part of a 3-year project whose objective is to create an entirely software + hardware controlled oil mill.

C 2007 Elsevier B.V. All rights reserved.
\end{abstract}

